Tropical Journal of Pharmaceutical Research February 2021; 20 (2): 249-256

ISSN: $1596-5996$ (print); 1596-9827 (electronic) (C) Pharmacotherapy Group, Faculty of Pharmacy, University of Benin, Benin City, 300001 Nigeria.

\title{
Effect of miR-384-targeting LINC00491 on proliferation, migration and invasion of tongue squamous cell carcinoma cells
}

\author{
Yun Deng ${ }^{1 *}$, Zhiwei Luo ${ }^{1}$, Peilin Feng ${ }^{2}$, Shuai Wang ${ }^{3}$ \\ ${ }^{1}$ Department of Stomatology, Chengdu Fifth People's Hospital, ${ }^{2}$ Department of Stomatology, Dayi County People's Hospital, \\ Chengdu 611300, ${ }^{3}$ Department of Stomatology, Jintang County People's Hospital, Chengdu 610400, PR China
}

*For correspondence: Email: Dengbinnews@126.com

Sent for review: 21 October 2020

Revised accepted: 27 January 2021

\begin{abstract}
Purpose: To investigate the effect of long-chain non-coding RNA LINC00491 (LncRNA LINC00491) on the proliferation, migration and invasion of tongue squamous cell carcinoma (TSCC) cells, and the underlying mechanism.

Methods: Real-time quantitative polymerase chain reaction (qRT-PCR) was applied to determine the expressions of LINC00491 and micro-RNA-384 (miR-384). Furthermore, LINC00491 and miR-384 were transfected into CAL-27 cells, while cell cycle was analyzed using flow cytometry. Cell proliferation was determined by methyl thiazolyl diphenyl-tetrazolium (MTT) assay. Cell migration and invasion were evaluated using Transwell experiments. The relationship between LINC00491 and miR-384 was confirmed using double luciferase reporting assay, while protein expression levels of P21, Ki67, Ecadherin, $N$-cadherin, and vimentin were assayed with Western blotting.

Results: The expression of LINC00491 increased in TSCC tissues $(p<0.05)$. The proportion of cells in G1-phase increased, while the proportion of cells in S-phase decreased $(p<0.05)$. There was decrease in cell survival, cell migration and cell invasion $(p<0.05)$. The protein expression levels of Ki67, $N$ cadherin, and vimentin were lowered, while those of $P 21$, E-cadherin protein were increased $(p<0.05)$. Transfection of LINC00491 and miR- 384 reduced the proportion of cells in G1 phase, but increased the proportion of cells in S-phase $(p<0.05)$. Moreover, cell survival, migration and invasion were increased. The protein expressions of Ki67, N-cadherin, and vimentin rose, while those of P21 and E-cadherin decreased $(p<0.05)$.

Conclusion: LINC00491 promotes the proliferation, migration and invasion of TSCC cells by inhibiting miR-384. This finding provides a potential target for the treatment of TSCC.
\end{abstract}

Keywords: LINC00491, MiR-384, Tongue squamous cell carcinoma, Proliferation, Migration, Invasion

This is an Open Access article that uses a fund-ing model which does not charge readers or their institutions for access and distributed under the terms of the Creative Commons Attribution License (http://creativecommons.org/licenses/by/4.0) and the Budapest Open Access Initiative (http://www.budapestopenaccessinitiative.org/read), which permit unrestricted use, distribution, and reproduction in any medium, provided the original work is properly credited.

Tropical Journal of Pharmaceutical Research is indexed by Science Citation Index (SciSearch), Scopus, International Pharmaceutical Abstract, Chemical Abstracts, Embase, Index Copernicus, EBSCO, African Index Medicus, JournalSeek, Journal Citation Reports/Science Edition, Directory of Open Access Journals (DOAJ), African Journal Online, Bioline International, Open-J-Gate and Pharmacy Abstracts

\section{INTRODUCTION}

Tongue squamous cell carcinoma (TSCC) is one of the common malignant tumors with high degree of metastasis. In recent years, the incidence of TSCC has significantly increased. At present, some progress has been made in the treatment of TSCC, but the 5-year survival of patients has decreased [1,2]. Therefore, it is important to understand the molecular pathways involved in the pathogenesis of TSCC. Long noncoding RNA (LncRNA) regulates cell 
proliferation, migration and invasion by binding to micro-RNA.

Previous studies have shown that LncRNA plays an important regulatory role in TSCC $[3,4]$. Longchain non-coding RNA LINC00491 (LncRNA LINC00491) is up-regulated, and promotes proliferation, migration and invasion of colon cancer cells [5]. However, the regulatory mechanism through which LINC00491 participates in the tumorigenesis of TSCC has not been elucidated. Bioinformatics analysis has shown that micro-RNA-384 (miR-384) may be the target gene of LINC00491. Studies have also shown that the expression of miR-384, which is low in pancreatic cancer, is involved in tumor cell proliferation [6]. However, it is not known whether LINC00491 participates in tumorigenesis of TSCC by regulating the expression of miR-384. The purpose of this study was to determine the effect of LINC00491 on the proliferation, migration and invasion of TSCC cells, and its regulatory effect on miR-384. The findings are expected to provide a theoretical foundation for the molecular mechanism involved in TSCC.

\section{EXPERIEMNTAL}

\section{Patients and reagents}

Thirty-eight TSCC patients treated in our hospital from March 2017 to December 2018 were enrolled in this study. The patients (28 males and 10 females) were pathologically confirmed as TSCC. The mean age of the patients was 58.54 \pm 6.57 years. The TSCC and corresponding adjacent tissues were obtained during surgery and kept cryopreserved at $-80^{\circ} \mathrm{C}$. This study was approved by the Ethics Committee of Chengdu Fifth People's Hospital. The included patients were informed in detail about the study, and each participant signed consent form in line with the National ethical guidelines for biomedical and health research involving human subjects [7].

Tongue squamous cell carcinoma cell line CAL27 was purchased from ATCC cell bank. Dulbecco's modified eagle medium (DMEM/F12), fetal bovine serum, Opti-MEM serumreducing medium, penicillin and streptomycin were bought from Gibco Company (USA). Trypsin and Lipofectamine 2000 were purchased from Thermo Fisher Company (USA). TRIzol was got from Invitrogen (USA), while qRT-PCR kits were supplied by TaKaRa Company in Japan.

Disordered meaningless negative sequence (siNC), LINC00491 small interfering RNA (siLINC00491), miR-384 oligonucleotide mimic (miR-384 mimics), negative control mimic NC sequence (miR-NC), and miR-384 specific oligonucleotide inhibitor (anti-miR-384) and its negative control (anti-miR-NC) were purchased from Shanghai Jima Pharmaceutical Technology Co. Ltd. Methylthiazolyl tetrazole (methylthiazolyl tetrazolium, MTT) was bought from Sigma Company, United States of America. Transwell chamber was obtained from Corning Company, United States. Matrigel matrix adhesive was purchased from BD Company, United States, while RIPA lysing buffer was obtained from Shanghai Lianmai Biological Engineering Co. Ltd. Bicinchoninic acid (BCA) protein quantitative detection kit, enhanced chemiluminescence reagent (electrochemiluminescence, ECL) and sodium dodecyl sulfate (SDS) were purchased from Beijing Full Gold Biotechnology Co. Ltd. Rabbit anti-human P21 and proliferating marker protein cell proliferating nucleus antigen-67 antibody (antigen identified by monoclonal antibody, Ki67) were supplied by American CST Company. Rabbit anti-human epithelial cadherin (E-cadherin), nerve-type cadherin (N-cadherin) and vimentin were got from American Santa Cruz company, while goat anti-rabbit second antibody labeled with horseradish peroxidase (HRP) were obtained from Abcam (USA).

\section{Cell transfection}

The CAL-27 cells were cultured in DMEM medium containing $10 \%$ fetal bovine serum, penicillin (100 $\mathrm{U} / \mathrm{mL}$ ) and streptomycin (100 $\mu \mathrm{g} / \mathrm{mL}$ ) in a $5 \% \mathrm{CO}_{2}$ incubator at $37^{\circ} \mathrm{C}$, and, the culture medium was changed every 2 days. When the cells grew to $80 \%$ fusion, they were digested with $0.25 \%$ trypsin, and the cell suspension seeded at a density of $1 \times 10^{5}$ cells/ $\mathrm{mL}$ in 96-well plates (100 $\mathrm{LL} /$ well $)$. When the cells grew to $70 \%$ fusion, the culture medium was changed to Opti-MEM medium. Plasmids loaded with si-NC, si-LINC00491, si-LINC00491 and anti-miR-NC, si-LINC00491 and anti-miR384 were transfected into CAL-27 cells, in line with the instructions of Lipofectamine 2000 reagent, and labeled as si-NC group, siLINC00491 group, si-LINC00491+anti-miR-NC group and si-LINC00491 + anti-miR-384 group, respectively. Six hours after transfection, the culture medium of all cells was replaced with complete DMEM containing $10 \%$ fetal bovine serum, and culturing was continued for $48 \mathrm{~h}$.

\section{Quantitative real-time PCR}

Total RNA was extracted from the frozen TSCC tissues, para-cancerous tissues and transfected CAL-27 cells using TRIzol method. The concentration of RNA was determined with nucleic acid and protein analyzer. The RNA 
samples were reverse-transcribed to cDNA using TransScript ®miRNA First-Strand cDNA Synthesis SuperMix reverse transcription kit, according to the kit manufacturer's instructions. The cDNAs were used as templates for qRT$\mathrm{PCR}$ with the primer sequences indicated in Table 1. The relative expressions of LINC00491 and miR-384 were calculated using the $2-\Delta \Delta \mathrm{Ct}$ method.

\section{Flow cytometry}

The CAL-27 cells in logarithmic growth phase were inoculated into 6 -well plates at a density of $3 \times 10^{4}$ cells $/ \mathrm{mL}(150 \mu \mathrm{L} /$ well $)$. When the cells grew to $50 \%$ fusion, cells in the transfection groups were fixed in $70 \%$ ethanol for $24 \mathrm{~h}$ and stained with propidium iodide (PI). The percentage of cells in each cell cycle phase (G1 phase, S phase, M phase) was determined and analyzed using flow cytometry.

\section{Determination of cell viability}

The CAL-27 cells in logarithmic growth phase were inoculated into 96-well plates (100 $\mu \mathrm{L} /$ well) at a density of $3 \times 10^{4}$ cells $/ \mathrm{mL}$. After $48 \mathrm{~h}$ cell transfection, $20 \mu \mathrm{L}$ of MTT solution $(5 \mathrm{mg} / \mathrm{mL}$ ) was added to each well. The plates were incubated at $4{ }^{\circ} \mathrm{C}$ for $4 \mathrm{~h}$, after which the medium was replaced with $150 \mu \mathrm{L}$ dimethyl sulfoxide (DMSO) to solubilize the formazan crystals formed. The absorbance of the formazan solution in each well was read at $490 \mathrm{~nm}$ in an enzyme labeling instrument. The readings were used to calculate cell viability.

\section{Evaluation of cell migration and invasion}

\section{Cell migration}

CAL-27 cells in logarithmic growth phase in each group were adjusted to a density of $5 \times 10^{4}$ cells $/ \mathrm{mL}$. The cell suspension was inoculated into the upper transwell chamber at a concentration of $200 \mu \mathrm{L} /$ well. Culture medium containing $10 \%$ fetal bovine serum was added to the lower chamber $(600 \mu \mathrm{L} /$ well $)$. The cells were cultured at $37{ }^{\circ} \mathrm{C}$ in a $5 \% \mathrm{CO}_{2}$ incubator for $24 \mathrm{~h}$. Then, the transwell chamber was washed twice with PBS, and non-migrated cells on the membrane were wiped off with a cotton swab. Then, the number of cells that passed through the membrane were counted under microscope and compared among the groups.

\section{Cell invasion}

$40 \mu \mathrm{L}$ of Matrigel solution was diluted with $400 \mu \mathrm{l}$ of DMEM medium without fetal bovine serum. Then, the diluted solution was added to the upper transwell chamber $(40 \mu \mathrm{L}$ per well), and incubated in a $37^{\circ} \mathrm{C}$ incubator for $5 \mathrm{~h}$. The subsequent steps were same as in the cell migration experiment. The number of invasive cells was observed under a light microscope.

\section{Double luciferase reporter assay}

StarBase prediction showed that LINC00491 contained nucleotide sequences complementary to miR-384. The binding site was mutated using gene mutation technology, and the sequence containing binding site and mutation site was inserted into luciferase reporter gene vector to construct wild type vector WT-LINC00491 and mutant vector MUT-LINC00491. The MiR-NC and miR-384 mimics were co-transfected into CAL-27 cells with WT-LINC00491 and MUTLINC00491, respectively, and cultured for $48 \mathrm{~h}$. The luciferase activity of each group of cells was determined.

\section{Statistical analysis}

Measured data in normal distribution are expressed as mean $\pm S D$. The data were analyzed using SPSS 21.0 statistical software. Independent sample $t$-test was used for comparison between two groups, while single factor analysis of variance was used for comparison amongst multiple groups. Statistical significance of difference was assumed at $p<$ 0.05 .

\section{RESULTS}

\section{Expression of LINC00491 in TSCC}

Compared with normal tissues, the expression level of LINC00491 in TSCC tissue was significantly increased $(p<0.05)$.

Table 1: The primer sequences used in qRT-PCR

\begin{tabular}{lll}
\hline Gene & $\mathbf{5}^{\prime} \mathbf{3}^{\prime}$ & $\mathbf{3}^{\prime} \mathbf{n}^{\prime}$ \\
\hline LINC00491 & CTTGTCTCCTCCCGTGAACT & GACCTTTGGCCTCTTTTGGG \\
miR-384 & ACATTTTCCGGAACCCTGTTG & TCTTGGACAAATGTTTCACGGA \\
GAPDH & AACGGATTTGGTCGTATTG & GGAAGATGGTGATGGGATT \\
U6 & GGAAGATGGTGATGGGATT & GGAACGCTTCACGAATTTG \\
\hline
\end{tabular}


Table 2: Relative expression levels of LINC00491 in TSCC

\begin{tabular}{lc}
\hline Group & $\begin{array}{c}\text { Relative expression } \\
\text { level of LINC00491 }\end{array}$ \\
\hline Normal tissue & $1.00 \pm 0.06$ \\
TSCC tissue & $2.69 \pm 0.08^{*}$ \\
$t$ & 50.700 \\
$P$ & 0.000 \\
\hline${ }^{*} P<0.05$, compared to the normal tissue
\end{tabular}

\section{LINC00491 inhibited the proliferation of CAL-} 27 cells

Compared with the si-NC group, the proportion of cells in the G1 phase of the si-LINC00491 group was significantly increased, while the proportion of cells in the $S$ phase was significantly reduced $(p<0.05)$. Cell survival was significantly reduced, and the Ki67 protein level was significantly decreased by LINC00491 $(p<0.05)$. However, the protein expression level of P21 was markedly increased $(p<0.05)$. These results are presented in Figure 1 and Table 3.

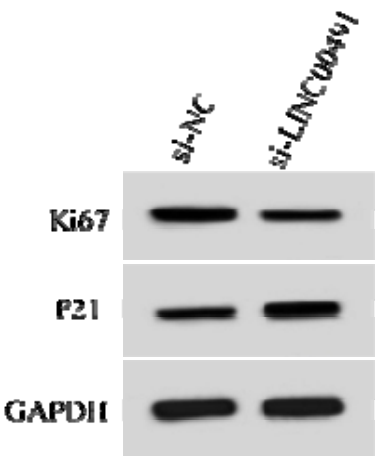

Figure 1: Effect of LINC00491 on the protein expressions of Ki67 and P21
Inhibitory effect of LINC00491 on migration and invasion of CAL-27 cells

Compared with the si-NC group, the numbers of migrated and invaded cells in the si-LINC00491 group were significantly reduced $(p<0.05)$. The expression level of E-cadherin protein was significantly increased, while the protein expression levels of $\mathrm{N}$-cadherin and vimentin were significantly reduced $(p<0.05)$. These results are shown in Figure 2 and Table 4.

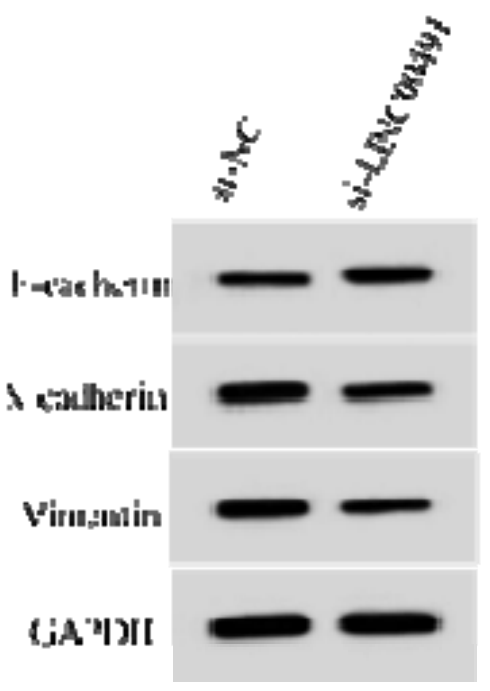

Figure 2: Protein expressions of E-cadherin, $\mathrm{N}$ cadherin and vimentin

\section{LINC00491 targets miR-384}

Using StarBase, it was predicted that there were binding sites between LINC00491 and miR-384, as shown in Figure 3.

Table 3: Inhibitory effect of LINC00491 on the proliferation of CAL-27 cells

\begin{tabular}{lllllll}
\hline Group & G1 & S & M & Survival & Ki67 & P21 \\
\hline si-NC & $33.19 \pm 3.11$ & $32.96 \pm 3.03$ & $33.85 \pm 3.21$ & $100.05 \pm 6.63$ & $0.67 \pm 0.05$ & $0.19 \pm 0.02$ \\
si-LINC00491 & $45.03 \pm 4.20^{*}$ & $21.17 \pm 2.59^{*}$ & $33.80 \pm 3.19$ & $52.29 \pm 4.33^{*}$ & $0.24 \pm 0.03^{*}$ & $0.58 \pm 0.04^{*}$ \\
$t$ & 6.797 & 8.873 & 0.033 & 18.094 & 22.123 & 26.162 \\
P-value & 0.000 & 0.000 & 0.974 & 0.000 & 0.000 & 0.000 \\
\hline
\end{tabular}

Data are presented as mean \pm SD $(n=9) .{ }^{*} P<0.05$, compared to the si-NC group

Table 4: Inhibitory effect of LINC00491 on migration and invasion of CAL-27 cells

\begin{tabular}{lcccccc}
\hline Group & LINC00491 & Migrated cells & Invaded cells & E-cadherin & N-cadherin & Vimentin \\
\hline si-NC & $1.00 \pm 0.05$ & $151 \pm 6.94$ & $92 \pm 4.28$ & $0.26 \pm 0.03$ & $0.81 \pm 0.06$ & $0.77 \pm 0.05$ \\
si-LINC00491 & $0.29 \pm 0.03^{*}$ & $75 \pm 4.20^{*}$ & $47 \pm 3.07^{*}$ & $0.78 \pm 0.05^{*}$ & $0.32 \pm 0.04^{*}$ & $0.25 \pm 0.03^{*}$ \\
$T$ & 36.529 & 28.107 & 25.630 & 26.754 & 20.385 & 26.754 \\
$P$ & 0.000 & 0.000 & 0.000 & 0.000 & 0.000 & 0.000 \\
\hline Data are expressed as mean \pm SD $(\mathrm{n}=9) .{ }^{*} P<0.05$, compared to the si-NC group &
\end{tabular}


The results of dual luciferase report assay demonstrated that co-transfection of cells with wild-type vector WT-LINC00491 led to significant reduction in the luciferase activity of the miR-384 group, when compared with that of the miR-NC group $(p<0.05)$. However, co-transfection with mutant vector MUT-LINC00491 did not cause any significant difference in luciferase activity between the miR-384 group and the miR-NC group ( $p>0.05$; Table 5$)$. In contrast, compared with the si-NC group, the expression level of miR-384 in cells of the si-LINC00491 group was significantly increased $(p<0.05)$.

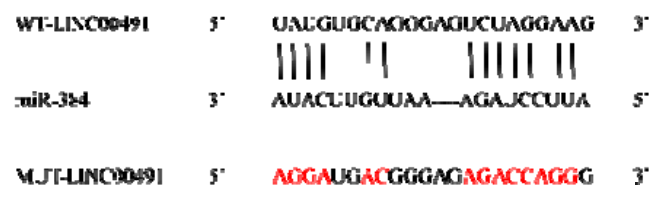

Figure 3: The binding sequence of LINC00491 and miR-384

Table 5: LINC00491 target miR-384

\begin{tabular}{lll}
\hline Group & WT-LINC00491 & MUT-LINC00491 \\
\hline miR-NC & $0.96 \pm 0.06$ & $0.95 \pm 0.06$ \\
miR-384 & $0.31 \pm 0.03^{*}$ & $0.98 \pm 0.07$ \\
$T$ & 29.069 & 0.976 \\
$P$-value & 0.000 & 0.344 \\
\hline
\end{tabular}

Data are expressed as mean \pm SD $(n=9) .{ }^{*} P<0.05$, compared to the miR-NC group

Table 6: LINC00491 regulates miR-384

\begin{tabular}{lc}
\hline Group & miR-384 \\
\hline si-NC & $0.98 \pm 0.06$ \\
si-LINC00491 & $3.67 \pm 0.09^{\#}$ \\
$T$ & 74.607 \\
$P$-value & 0.000 \\
\hline
\end{tabular}

Data are expressed as mean \pm SD $(\mathrm{n}=9)$. ${ }^{*} P<0.05$, compared to the miR-NC group

Inhibition of miR-384 expression enhanced the effect of LINC00491 on proliferation of CAL-27

Compared with the si-LINC00491+anti-miR-NC group, the proportion of $\mathrm{G} 1$ phase cells in the siLINC00491+ anti-miR-384 group was significantly reduced, while the proportion of $S$ phase cells was significantly increased $(p<$ $0.05)$. The survival of CAL-27 cells and the protein expression level of Ki67 were significantly increased, while the protein expression level of P21 was significantly decreased $(p<0.05)$. These results are shown in Figure 4 and Table 6.

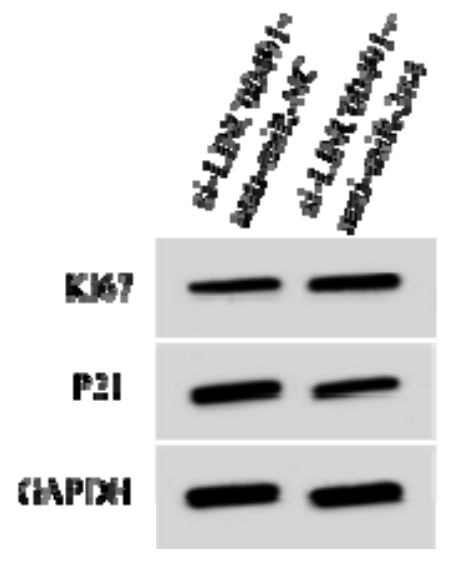

Figure 4: The expression level of Ki67 and P21 protein

\section{Inhibition of miR-384 expression enhanced the effect of LINC00491 on the migration and invasion of CAL-27 cells}

Compared with the si-LINC00491 + anti-miR-NC group, the numbers of migrated and invaded cells in the si-LINC00491 + anti-miR-384 group were significantly increased $(p<0.05)$. Western blot assay revealed that the protein level of $E$ cadherin was significantly reduced, while those of $\mathrm{N}$-cadherin and vimentin were significantly increased $(p<0.05)$. These results are presented in Figure 5 and Table 8.

\section{DISCUSSION}

The early symptoms of TSCC are not readily noticeable. Thus, most patients are in the middle and advanced stages of the disease at the point of diagnosis.

Table 7: Inhibition of miR-384 expression enhanced the effect of LINC00491 on the proliferation of CAL-27

\begin{tabular}{lllllll}
\hline Group & G1 & S & M & Survival & Ki67 & P21 \\
\hline $\begin{array}{l}\text { si-LINC00491+ } \\
\text { anti-miR-NC }\end{array}$ & $44.95 \pm 4.16$ & $21.21 \pm 2.62$ & $33.84 \pm 3.14$ & $52.32 \pm 4.38$ & $0.26 \pm 0.03$ & $0.57 \pm 0.04$ \\
si-LINC00491+ & $36.91 \pm 3.43^{*}$ & $28.96 \pm 2.91^{*}$ & $34.13 \pm 3.17$ & $89.10 \pm 5.54^{*}$ & $0.57 \pm 0.05^{*}$ & $0.26 \pm 0.02^{*}$ \\
anti-miR-384 & & & & & & \\
$T$ & 4.474 & 5.938 & 0.195 & 15.624 & 15.949 & 20.795 \\
$P$ & 0.000 & 0.000 & 0.848 & 0.000 & 0.000 & 0.000 \\
\hline
\end{tabular}

Results are expressed as mean \pm SD $(n=9)$. Data are expressed as mean \pm SD $(n=9) .{ }^{*} P<0.05$, compared to the miR-NC group 
Table 8: Inhibition of miR-384 expression enhanced the effect of LINC00491 on the migration and invasion of CAL-27 cells

\begin{tabular}{lllllll}
\hline Group & miR-384 & Migrated cells & Invaded cells & E-cadherin & N-cadherin & Vimentin \\
\hline $\begin{array}{l}\text { Si-LINC00491+ } \\
\text { anti-miR-NC }\end{array}$ & $1.01 \pm 0.06$ & $76 \pm 4.22$ & $48 \pm 3.09$ & $0.77 \pm 0.05$ & $0.34 \pm 0.04$ & $0.26 \pm 0.03$ \\
si-LINC00491+ & $0.24 \pm 0.03^{*}$ & $128 \pm 6.13^{*}$ & $79 \pm 3.87^{*}$ & $0.34 \pm 0.03^{*}$ & $0.70 \pm 0.05^{*}$ & $0.62 \pm 0.05^{*}$ \\
anti-miR-384 & & & & & & \\
$T$ & 34.435 & 20.962 & 18.779 & 22.123 & 16.867 & 18.522 \\
$P$-value & 0.000 & 0.000 & 0.000 & 0.000 & 0.000 & 0.000 \\
\hline${ }^{*} P<0.05$, compared to the si-LINC00491 + anti-miR-NC group & & &
\end{tabular}

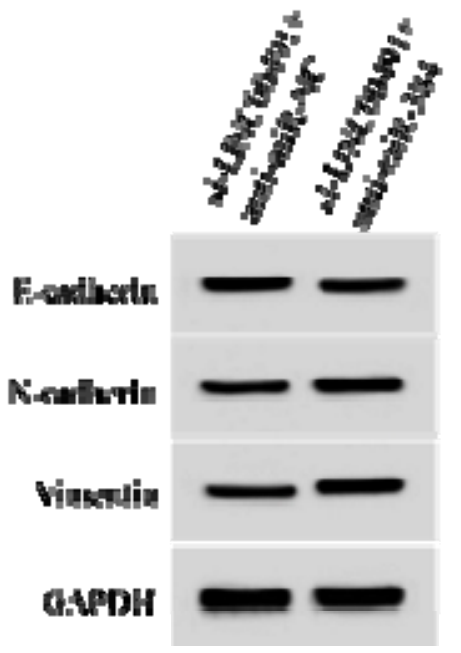

Figure 5: Protein expression levels of E-cadherin, Ncadherin and vimentin

At present, the mechanism involved in the pathogenesis of TSCC has not been fully elucidated. An understanding of the molecular mechanism underlying the pathogenesis of TSCC and its metastasis is of great significance for targeted therapy and improvement of the prognosis of patients. Previous studies have shown that LncRNA participates in biological processes such as proliferation, migration and invasion of TSCC cells by regulating the molecular axis of miRNA-target gene [8-10]. However, some aspects of the mechanisms associated with the involvement of LncRNA in the occurrence of TSCC have not been fully elucidated.

Studies have shown that LncRNA LINC00491 is highly expressed in lung squamous cell carcinoma, endometrial carcinoma, colon cancer and other malignant tumors, and may be used as an important index for cancer diagnosis and prognosis evaluation in patients [11-13]. The results of this study showed that the expression of LINC00491 was up-regulated in TSCC carcinoma. Similar results were obtained in previous studies, suggesting that LINC00491 plays an oncogenic role in the development of
TSCC. In this study, the expression of LINC00491 was inhibited, and the results showed significant suppression of the proliferation, migration and invasion of CAL-27 cells. These results suggest that inhibition of LINC00491 expression may block the proliferation, migration and invasion of TSCC cells, and induce cell cycle arrest in G1 phase.

In order to investigate the possible mechanism involved in the effect of LINC00491 on the proliferation, migration and invasion of TSCC cells, the expressions of proteins associated with proliferation, migration and invasion were assayed with Western blotting. The results showed that the expressions of P21 and Ecadherin were up-regulated in TSCC cells after inhibition of LINC00491 expression, while the expressions of $\mathrm{Ki67}, \mathrm{N}$-cadherin and vimentin were down-regulated. Studies have shown that Ki67 is highly expressed in tumors: it promotes cell proliferation, while P21 negatively regulates cell cycle and inhibits cell proliferation $[14,15]$.

Epithelial-mesenchymal transition (EMT) is closely related to tumor cell migration and invasion. The up-regulation of the expression of epithelial-type EMT inhibits the transformation of EMI and inhibits cell metastasis, while $\mathrm{N}$ cadherin and vimentin are metastasis-promoting stroma-type markers which are highly expressed in tumors [16]. The results of the present study suggest that the inhibition of LINC00491 expression may block the proliferation, migration and invasion of TSCC cells by regulating the expressions of P21, Ki67, E-cadherin, Ncadherin and vimentin, and inducing cell cycle arrest.

Moreover, the molecular mechanism involved in the effect of LINC00491 on the proliferation, migration and invasion of TSCC cells was investigated using double luciferase reporter assay and qRT-PCR assay. The results obtained confirmed that LINC00491 targeted binding of miR-384, and negatively regulated the expression of miR-384. Studies have shown that miR-384 targets metadherin and inhibits the 
growth, migration and invasion of gastric cancer cells [17]. It has been reported that LncRNA SNHG3 promotes the proliferation and invasion of breast cancer cells by regulating the expression of miR-384 [18]. In addition, LncRNA SNHG3 promotes the proliferation and migration of laryngeal cancer cells by regulating the molecular axis of miR-384-WEE1 [19].

It is known that miR-384 acts as a tumor suppressor gene in tumorigenesis. In order to find out whether LINC00491 affected the proliferation, migration and invasion of TSCC cells by regulating the expression of miR-384, siLINC00491 and anti-miR-384 were cotransfected into $\mathrm{CAL}-27$ cells. The results showed that the proportion of cells in G1 phase decreased, while the proportion of cells in $S$ phase increased, and there were marked increases in cell proliferation, migration and invasion. Moreover, the expressions of Ki67, Ncadherin and vimentin were up-regulated, while the expression of P21 and E-cadherin were down-regulated. Inhibition of miR-384 expression significantly weakened the inhibitory effect of LINC00491 on the proliferation, migration and invasion of CAL-27 cells. Thus, the inhibition of the expression of LINC00491 may suppress the proliferation, migration and invasion of TSCC cells and induce cell cycle arrest by up-regulating the expression of miR-384.

\section{CONCLUSION}

This study has demonstrated that LINC00491 is highly expressed in TSCC cells through a mechanism most likely associated with targeted regulation of miR-384 expression and protein expressions of P21, Ki67, E-cadherin, Ncadherin and vimentin. This finding may provide a potential basis for targeted therapy of TSCC.

\section{DECLARATIONS}

\section{Conflict of interest}

No conflict of interest is associated with this work.

\section{Contribution of authors}

We declare that this work was done by the authors named in this article, and all liabilities pertaining to claims relating to the content of this article will be borne by the authors. All authors read and approved the manuscript for publication. Yun Deng and Zhiwei Luo conceived and designed the study. Peilin Luo and Shuai
Wang collected and analyzed the data, while Bin Deng wrote the manuscript.

\section{Open Access}

This is an Open Access article that uses a funding model which does not charge readers or their institutions for access and distributed under the terms of the Creative Commons Attribution License (http://creativecommons.org/licenses/by/ 4.0) and the Budapest Open Access Initiative (http://www.budapestopenaccessinitiative.org/rea d), which permit unrestricted use, distribution, and reproduction in any medium, provided the original work is properly credited.

\section{REFERENCES}

1. Ng JH, lyer NG, Tan MH, Edgren G. Changing epidemiology of oral squamous cell carcinoma of the tongue: A global study. Head Neck 2017; 39(2): 297304.

2. Shibahara T. [Oral cancer-diagnosis and therapy]. Clin Calcium 2017; 27(10): 1427-1433.

3. Zheng Y, Zheng B, Meng X, Yan Y, He J, Liu Y. LncRNA $D A N C R$ promotes the proliferation, migration, and invasion of tongue squamous cell carcinoma cells through miR-135a-5p/KLF8 axis. Cancer Cell Int 2019; 19(1): 302-312.

4. Chen Y, Guo Y, Yan W. IncRNA RP5-916L7.2 correlates with advanced tumor stage, and promotes cells proliferation while inhibits cells apoptosis through targeting miR-328 and miR-939 in tongue squamous cell carcinoma. Clin Biochem 2019; 67(1): 24-32.

5. Wan J, Deng $D$, Wang $X$, Wang $X$, Jiang $S$, Cui $R$. LINC00491 as a new molecular marker can promote the proliferation, migration and invasion of colon adenocarcinoma cells. Onco Targets Ther 2019; 12(1): 6471-6480.

6. Wang G, Pan J, Zhang L, Wei Y, Wang C. Long noncoding RNA CRNDE sponges miR-384 to promote proliferation and metastasis of pancreatic cancer cells through upregulating IRS1. Cell Prolif 2017; 50(6): e12389.

7. Mathur R, Swaminathan S. National ethical guidelines for biomedical \& health research involving human participants, 2017: A commentary. Indian J Med Res 2018; 148(3): 279-283.

8. Chen $X, X u H$, Sun G, Zhang Y. LncRNA CASC9 Affects Cell Proliferation, Migration, and Invasion of Tongue Squamous cell Carcinoma via Regulating miR-4235p/SOX12 Axes. Cancer Manag Res 2020; 12(1): 277287.

9. Xiong L, Tang Y, Tang J, Wang X. Downregulation of IncRNA HOTTIP Suppresses the Proliferation, Migration, and Invasion of Oral Tongue Squamous Cell Carcinoma by Regulation of HMGA2-Mediated Wnt/ $\beta$ - 
Catenin Pathway. Cancer Biother Radiopharm 2020; 8(1): $1-10$

10. Wu X, Ma J, Chen J, Huang H. LncRNA CACS15 regulates tongue squamous cell carcinoma cell behaviors and predicts survival. BMC Oral Health 2019; 19(1): 231-241.

11. Liu J, Yao Y, Hu Z, Zhou H, Zhong M. Transcriptional profiling of long-intergenic noncoding RNAs in lung squamous cell carcinoma and its value in diagnosis and prognosis. Mol Genet Genomic Med 2019; 7(12): e994e1004.

12. Xia L, Wang Y, Meng Q, Su X, Shen J, Wang J, He H, Wen $B$, Zhang $C, X u \quad M$. Integrated Bioinformatic Analysis of a Competing Endogenous RNA Network Reveals a Prognostic Signature in Endometrial Cancer. Front Oncol 2019; 9(1): 448-458.

13. Wang WJ, Li HT, Yu JP, Han XP, Xu ZP, Li YM, Jiao ZY, Liu HB. A Competing Endogenous RNA Network Reveals Novel Potential IncRNA, miRNA, and mRNA Biomarkers in the Prognosis of Human Colon Adenocarcinoma. J Surg Res 2019; 235(1): 22-33.
14. Yang C, Zhang J, Ding M, Xu K, Li L, Mao L, Zheng J. $K i 67$ targeted strategies for cancer therapy. Clin Transl Oncol 2018; 20(5): 570-575.

15. Kreis NN, Louwen F, Yuan J. The Multifaceted p21 (Cip1/Waf1/CDKN1A) in Cell Differentiation, Migration and Cancer Therapy. Cancers (Basel) 2019; 11(9): 1220.

16. Odero-Marah V, Hawsawi O, Henderson V, Sweeney J. Epithelial-Mesenchymal Transition (EMT) and Prostate Cancer. Adv Exp Med Biol 2018; 1095:101-110

17. Wang F. miR-384 targets metadherin gene to suppress growth, migration, and invasion of gastric cancer cells. J Int Med Res 2019; 47(2): 926-935.

18. Ma Q, Qi X, Lin X, Li L, Chen L, Hu W. LncRNA SNHG3 promotes cell proliferation and invasion through the miR-384/hepatoma-derived growth factor axis in breast cancer. Hum Cell 2020; 33(1): 232-242.

19. Wang L, Su K, Wu H, Li J, Song D. LncRNA SNHG3 regulates laryngeal carcinoma proliferation and migration by modulating the miR-384/WEE1 axis. Life Sci 2019; 232(1): 116597-116607. 